\title{
CONSIDERACIONES SOBRE EDUCACIÓN, INTERCULTURALIDAD Y LA PERSPECTIVA DE-COLONIAL
}

\author{
Óscar Julián Cuesta Moreno ${ }^{58}$
}

\begin{abstract}
RESUMEN
El presente escrito reflexiona sobre el pensamiento pedagógico propio en América Latina desde la perspectiva de-colonial de algunos autores suramericanos. En una primera parte explora sucintamente las bases de dicha perspectiva, especialmente centrando la crítica en la modernidad europea que ha perpetuado una notable hegemonía; posteriormente, recupera la propuesta de la educación intercultural como un aporte a la constitución de un pensamiento educativo propiamente latinoamericano; y finalmente, trae a colación algunas consideraciones sobre el currículo bajo la mirada de la de-colonialidad.
\end{abstract}

Palabras clave: modernidad, colonialidad, de-colonialidad, pensamiento pedagógico, interculturalidad y currículo.

\begin{abstract}
The present paper reflects on the pedagogic thought proper to Latin America from the decolonial perspective of some South American authors. Initiating, it explores closely the bases of this perspective, specially centering the critic in the modernization of Europe that has perpetuated a notable hegemony; subsequently, recuperates the proposal of intercultural education as a contribution to the constitution of a properly Latin-American educational thought; and finally, brings to coalition some considerations about the curriculum under the sight of the de-coloniality.
\end{abstract}

Key words: modernity, colonial, of - colonial, pedagogic thought, intercultural and curriculum.

Recibido: 19 de abril de 2010 Aceptado: 24 de mayo de 2010

\section{INTRODUCCIÓN}

En los libros enciclopédicos y escolares de historia se muestra con gran pompa y detalle la historia europea y norteamericana de los últimos siglos, haciendo hincapié en los avances científicos, las teorías sociales y los pensadores que alimentaron los principios e ideales de la ilustración, la revolución francesa, la independencia norteamericana y la revolución industrial.

58 Docente Universidad Santo Tomás. Candidato a Magister en educación de la Universidad Pedagógica Nacional. Comunicador Social de la Universidad Santo Tomás. Técnico en locución y producción de medios audiovisuales del Colegio Superior de Telecomunicaciones y Especialista en docencia universitaria de la Universidad Cooperativa de Colombia. Se desempeña como docente universitario y educador en proyectos con población vulnerable. E-mail: oscarcuesta@colombia.com 
En una notable desigualdad, hablando en términos cualitativos y cuantitativos, la historia de los nativos americanos, de su violenta conquista y explotación, del trato abyecto a los africanos raptados de sus tierras y esclavizados por varias generaciones, es tratada en menos páginas e ilustrada con pocos retratos.

El trato conspicuo y benévolo a la modernidad europea ${ }^{59}$ en dichos libros de no es gratuito; así como tampoco es gratuito que la sabiduría y cosmovisión de los pueblos americanos y afro-descendientes sean explicadas de manera anecdótica y curiosa, restándole toda validez filosófica y epistemológica. La historia contada así, evidentemente, educa a los niños y jóvenes con una perspectiva deliberadamente dependiente del modelo europeo y, por si fuera poco, motivando una silenciosa vergüenza sobre su pasado ancestral.

\section{Consideraciones iniciales}

Varios pensadores latinoamericanos desde la década del sesenta se han preguntado por esta inequitativa forma de contar la historia y de enseñarla (por nombrar algunos: Eduardo Galeano, Orlando Fals Borda, Enrique Dussel, Darcy Riveiro). En el último tiempo, esa preocupación va más allá y los intelectuales e investigadores han hecho notorios esfuerzos para revalidar la producción de conocimiento propio de los pueblos latinoamericanos, criticando la hegemonía europea y norteamericana y sus principios de absolutos de verdad y protocolos científicos para alcanzarla (algunos autores de esta perspectiva son: Enrique Dussel, Aníbal Quijano, Arturo Escobar, Walter Mignolo, Edgar Lander, Santiago Castro-Gómez, Catherine Walsh, Cristian Rojas).

En ese sentido, los nuevos enfoques que indagan sobre la modernidad, la colonialidad y decolonialidad, buscan una reflexión constante sobre la realidad cultural y política latinoamericana, que incluye el conocimiento subalternizado de los grupos explotados y oprimidos. Así mismo, esta nueva mirada "debe ser entendida como una manera diferente del pensamiento, en contravía de las grandes narrativas modernistas — la cristiandad, el liberalismo y el marxismo - , localizando su propio cuestionamiento en los bordes mismos de los sistemas de pensamiento e investigaciones hacia la posibilidad de modos de pensamiento no-eurocéntricos" (Escobar, 2003, p. 54).

Para continuar, es pertinente decir que la modernidad establece un orden que, según Escobar (2003), está basado en la razón, el individuo, el conocimiento experto y los mecanismos administrativos ligados al Estado, de allí que una reflexión y revisión crítica de dicha hegemonía tenga que cruzar por estos linderos, que tienen sus cimientos en el supuesto que el mundo puede ser conocido y la naturaleza controlada. ${ }^{60}$

59 La modernidad, vista con benevolencia, formuló ideas y pensamientos que han mejorado la forma de organizar la sociedad y alcanzar conocimientos; sin embargo, sus concepciones se han extendido gracias a la hegemonía de unas pocas naciones explotadoras que han sacado provecho de su situación histórica, manteniendo una dependencia de los pueblos hacia sus formas de producción; de esta manera Europa queda en la cima y los otros países en puestos terciarios.

60 La modernidad, producto de un modo de acercarse al conocimiento, de organizase socialmente y de estructurar una particular forma de producción económica, es un orden puramente europeo; sin embargo, a través de los procesos de dominación y globalización se ha extendido por el mundo como forma única de constituirse, negando las alternativas brindadas por las cosmovisiones de otros pueblos. 
De allí la importancia de buscar alternativas al paradigma occidental europeo, evitando reproducir las formas, visones y verdades de tal modelo hegemónico, como se ha venido haciendo desde la independencia, donde en lugar de establecer la autonomía cultural para América Latina, se constituyó un cordón umbilical que ha mantenido a los países conquistados en una incuestionable dependencia. Las alternativas, en palabras de Mignolo (2001), deben originarse en la misma forma de buscar y producir el conocimiento, pues se necesita un pensamiento de frontera que enfrente el colonialismo de la epistemología occidental desde la perspectiva de las fuerzas epistémicas que han sido convertidas en formas subalternas del conocimiento (comúnmente llamadas folclor, mito y leyenda).

Precisamente, hablando de esa forma epistemológica alternativa a los cánones del cientifismo europeo, que al mismo tiempo es bondadoso y nefasto (crea vacunas e inventa armas biológicas), Walsh (2007) se pregunta si el conocimiento sólo puede ser producido en la academia y bajo los parámetros occidentales. A lo que la investigadora responde que, efectivamente, las ciencias sociales pueden y deben ser repensadas en una pluriversatilidad epistemológica que tenga en cuenta y, sobre todo, dialogue con las formas de conocimiento extra-académicas y extra-científicas. ${ }^{61}$

El pensamiento europeo y los protocolos que reglamentan su producción (metodologías y concepción de verdad), responden a "formulaciones teóricas monolíticas, monoculturales y 'universales' y que posicionan el conocimiento científico occidental como central, negando así o relegando al estatus de no conocimiento, a los saberes derivados de lugar y producidos a partir de relaciones sociales y culturales distintas" (Walsh, 2007, p. 103).

Ese eurocentrismo epistemológico, que algunos autores llaman colonialidad de saber, estableció una perspectiva única del conocimiento que descartó la producción intelectual indígena y afroamericana como conocimiento. Que, desde el punto de vista pedagógico, también negó las formas y prácticas particulares de estos grupos para transmitir saberes y formar sujetos, es decir, de educar.

\section{Hacia un pensamiento pedagógico propio desde la perspectiva de-colonial}

La hegemonía europea y norteamericana está tan arraigada en el sistema escolar, que los contenidos ofrecidos en los libros de historia anteriormente mencionados, han logrado establecer una identidad vergonzosa en los sujetos latinoamericanos, quienes se consideran a sí mismos inferiores y a ellos (europeo blanco) los estiman como superiores. Basta con preguntar a un muchacho a quién le cree más, si a un artículo publicado en una revista inglesa o uno de una revista local. De igual manera, ocurre con algunos investigadores, quienes se

61 De lo que se trata, según Walsh (2007, p. 104), es de "refutar los supuestos que localizan la producción de conocimiento únicamente en la academia, entre académicos y dentro del cientificismo, los cánones y los paradigmas establecidos. También refutar las concepciones de racionalidad que rigen el conocimiento mal llamado 'experto', negador y detractor de las prácticas, agentes y saberes que no caben dentro de la racionalidad hegemónica y dominante". 
sientan más orgullosos si su escrito es divulgado por una editorial norteamericana que por una colombiana.

Las elites de los países latinoamericanos, albergadas por un afán de llegar a ser como sus antepasados europeos, han establecido un sistema escolar que responde a su frustración: en Colombia, por ejemplo, se han traído misiones alemanas, francesas y españolas para organizar la educación conforme con los cánones hegemónicos y euro céntricos de la modernidad, para que los nuevos sujetos de la nación colombiana piensen, hablen y vean el mundo según dicha visión. Dejando de lado, desde luego, el saber indígena y afro-descendiente, pueblos que son invisibles de por sí en los contendidos de la historia oficial.

El conocimiento de estos pueblos, tildado de mito y leyenda, debe ser parte de un despertar pedagógico de un nuevo currículo, pues alimenta la verdadera construcción de una nación pluriculturar y multiétnica como la colombiana. Esto implicaría, trayendo a colación las palabras de Walsh (2007), comprender, actuar e imaginar el mundo de otra manera, distinta a los cánones euro céntricos.

Claramente, la perspectiva de-colonial implica una reflexión profunda de las instituciones educativas, dado que mantienen esquemas y formas de la modernidad, los cuales responden a circunstancias de otro momento histórico, con otros intereses políticos y culturales, distintos a los que necesita una sociedad contemporánea (Tiramonti, 2005), que exige un desprendimiento de la dependencia científica y económica de la hegemonía europea.

Lo ideal sería constituir una educación colombiana que revalide las diferencias en las estructuras coloniales del poder, del saber y del ser, logrando un proceso y proyecto que re-conceptualice y refunde las estructuras sociales, epistémicas y de existencias que ponen en escena y en relación equitativa lógicas, prácticas y modos culturales diversos de pensar y vivir (Walsh, 2006).

Aunque no existe como tal una propuesta pedagógica de-colonial para la práctica educativa, las reflexiones entorno a la epistemología y a la revalidación de los conocimientos no occidentales y alternativos, implican, de hecho, un debate en los cimientos mismos de la pedagogía, puesto que incursionan en los principios de la producción del mismo conocimiento.

La pedagogía, en líneas generales, hace referencia a la reflexión sobre la práctica educativa, es decir, a la manera en que son formados los sujetos de la sociedad. Establecer una revisión crítica a la manera como han sido invisibilizadas ciertas culturas en pro de mantener una dependencia a la producción intelectual hegemónica, brinda las luces de un pensamiento pedagógico propio para Latinoamérica, puesto que sienta las bases de un programa educativo particular a la cosmovisión local e independiente a la estructura colonial.

Los pueblos indígenas y afro-descendientes poseen formas y metodologías, para formar a sus niños y jóvenes, que deben ser escuchadas, pues responden a una sabiduría inveterada 
que propende por mantener una relación sana con el otro y con la naturaleza, contrario a la relación belicosa e individualista de la sociedad capitalista establecida por la modernidad.

Para ejemplificar esto, vale la pena citar el siguiente caso. En una reunión realizada en la Universidad Santo Tomás de Bogotá, una Taita Kogi sentaba la siguiente diferencia: cuando un miembro de nuestra familia es visto robando algo, se le lleva a donde los ancianos, quienes le preguntan de quién aprendió a robar o de dónde tomó ese ejemplo, así llegan a determinar dónde empiezan los malos comportamientos. En su sociedad, cuando alguien es visto robando se le castiga y nunca se sabe el por qué de los comportamientos, sin olvidar que nunca se escucha a los ancianos.

\section{Interculturalidad en la educación}

Una propuesta novedosa realizada en el Perú por Walsh (2000), muestra que es posible empezar a establecer un contenido escolar que reconozca los saberes de los pueblos indígenas, afro-descendientes y mestizos. Esta iniciativa tiene como pilar reconocer la interculturalidad en la educación. El presente artículo considera que esta iniciativa es una primera invitación para empezar a consolidar un pensamiento pedagógico propio en Latinoamérica desde la perspectiva de-colonial.

Antes de continuar, se debe entender interculturalidad como un intercambio cultural que se establece en términos equitativos y en condiciones de igualdad. A diferencia de la multiculturalidad, que es un término que describe la existencia de varias culturas pero no las relaciones entre ellas, y de la pluriculturalidad, que habla solamente sobre la existencias y convivencia de varias culturas en un territorio, la interculturalidad implica intercambio y articulación entre personas y grupos culturales (Walsh, 2000).

Walsh (2000, p. 12) platea que, la interculturalidad, motiva un espacio fronterizo de relación y negociación que también se construye y del cual emergen "nuevas estrategias, expresiones, iniciativas, sentidos y prácticas (inter) culturales que desafían la homogeneidad, el control cultural, y la hegemonía de la cultura dominante".

Como se ha venido afirmando, el sistema educativo se convierte en un escenario vital para poner en juego y desarrollar el nuevo pensamiento de-colonial, empezando por el establecimiento de una dinámica intercultural, puesto que es la base para consolidar la constitución de sujetos consientes de la historia desigual a la que a apostado la modernidad y, sobre todo, conscientes de los conocimientos y prácticas culturales de los pueblos de su continente.

Lo anterior implica un sistema educativo donde no se aprenda la historia vista por los historiadores de accidente, donde los estudiantes no tengan que aprender los saberes, principios, costumbres y pensamientos de los personajes europeos y norteamericanos, sino que tengan que empezar a descubrir y entender la historia y sabiduría de los pueblos latinoamericanos. 
En ese orden de ideas, para Walsh (2000) la educación intercultural se enriquece con la construcción de una escuela donde los profesores sean indígenas, afros, mestizos, hispanos y extranjeros. Aunque es una posición idealista para las actuales circunstancias, es necesario imaginar una escuela donde se encuentren y dialoguen las diferentes cosmovisiones que hacen de Colombia y, por extensión de América Latina, una gran nación colmada de diversidad.

Una educación intercultural "busca contribuir a una interacción respetuosa y fecunda entre individuos y culturas, poniendo énfasis en el reconocimiento, respeto y atención pedagógica de las diferencias y la diversidad cultural en todos los niveles, modalidades y aspectos del sistema educativo y en relación a cualquier campo del conocimiento" (Walsh, 2000, p. 18).

Así pues, la interculturalidad requiere una innovación pedagógica y curricular, que tenga en cuenta contenidos y experiencias culturales y, de manera preponderante, procesos de interacción social, que permitan construir (como se ha dicho) un conocimiento alternativo y no colonial, basado en el diálogo, el reconocimiento y la compresión del otro.

Aunque las propuestas etnoeducativas han puesto en discusión dinámicas interesantes, la interculturalidad educativa implica un complejidad mayor, pues la primera enfatiza en la enseñanza y aprendizaje de lo propio, y esta última "intenta promover una relación comunicativa y crítica entre seres y grupos distintos, y también extender esa relación en la tarea de construir sociedades realmente plurales y equitativas" (Walsh, 2000, p. 27).

La iniciativa intercultural para el Perú implica una formación en investigación que procura involucrar al alumno en procesos de descubrir y construir conocimiento, con el fin de que ese saber transforme socialmente. Asimismo, intenta que los docentes sean agentes interculturales que, por su experiencia misma, poseen un repertorio cultural propio que deben entender y abrir a los demás.

Walsh (2000) enuncia tres criterios para la interculturalidad en el aula: tener presente el contexto sociocultural del centro escolar; segundo, observar la realidad sociocultural de los alumnos y sus familias; tercero, alimentar el perfil de los docentes y sus relaciones con la comunidad de los alumnos y sus familias.

Así mismo, es pertinente recordar los criterios pedagógicos plateados por la investigadora para el desarrollo intercultural: autoestima y reconocimiento de lo propio; los conocimientos, los saberes y las prácticas locales; la identificación y el reconocimiento de las diferencias y de la otredad; conocimientos y prácticas de los otros; problemáticas de conflictos culturales, racismo y relaciones culturales negativas; unidad y diversidad; y la comunicación, interrelación y cooperación.

Como se puede observar, la propuesta intercultural para la educación articula principios que no son ajenos a los postulados y críticas de los autores latinoamericanos que investigan sobre la modernidad, la colonialidad y de-colonialidad. En especial, porque parte de reconocer el conocimiento de las culturas invisibilizadas por la hegemonía europea $\mathrm{y}$, además, porque 
motiva a investigar y contar la historia de otra forma, alimentando el giro epistemológico que pretende revisar los postulados científicos de la producción del saber.

\section{A propósito de un currículo de-colonial}

Es propicio, para terminar este artículo, realizar unas reflexiones acerca del currículo bajo la perspectiva de-colonial, el cual debe partir, primero que todo, de entender que la escuela contemporánea ha mercantilizado la educación, convirtiendo la producción simbólica propia del saber cultural en una mercancía.

Para Tadeu da Silva (1997), el currículo debe ser visto como un dispositivo histórico y social. Esto se puede observar en Latinoamérica a partir de los contenidos expuestos y las formas de transmitir dichos contenidos en las escuelas del continente. Se ha privilegiado la visión euro céntrica y ocultado el conocimiento indoamericano y afro-descendiente. Un ejemplo tangible es el siguiente: el 12 de octubre se celebra el descubrimiento de América; un currículo crítico vería tal fecha con un color algo menos efusivo: el inicio de la conquista.

Un programa para una escuela de-colonial, inevitablemente debe observar que los currículos son producidos y creados a través de relaciones sociales entre los grupos interesados (Tadeu da Silva, 1997), no son producto de iniciativas originadas en despachos ministeriales o en oficinas de rectorías escolares.

En ese sentido, el currículo, tal y como lo platea la crítica a la colonialidad, debe dar cuenta de las relaciones de clase, de género y raza en el marco de un globalización que pretende homogeneizar los mercados y extender la modernidad hegemónica europea a todo el mundo.

Un programa de-colonial, igualmente, entiende que las dinámicas sociales no son aisladas: "el privilegio y confort de ciertos individuos, grupos y naciones está indisociablemente unido a la privación y al sufrimiento de otros individuos, otros grupos y otras naciones" (Tadeu da Silva, 1997). En otras palabras, que la riqueza y pompa europea y norteamericana no sería posible sin la subyugación y explotación de los países de la periferia. Seguramente, un estudiante consciente de esto consumiría de manera diferente los productos de las transnacionales y dejaría de envidiar con la misma intensidad el modo de vida de las naciones hegemónicas.

Tadeu da Silva (1997) propone una primera iniciativa para de-colonizar el currículo: "interrumpir, subvertir y desestabilizar los significados y representaciones existentes en las llamadas fechas conmemorativas", dado que estas "condensan en su estructura y espíritu precisamente aquella relación voyeurística que los grupos dominantes mantienen con los grupos subordinados". Donde aquellos, desde arriba, miran con contemplación y arrogancia la celebración de esas fechas que termina "reforzando una relación neocolonialista con grupos socialmente subordinados", donde el otro se torna exótico. 
Este currículo de-colonial, igualmente, propende por una construcción social del mismo, donde se involucren todos los intereses sociales, de tal manera que se logre una construcción verdaderamente social del conocimiento, como la propuesta en la educación intercultural. Se trata, pues, de terminar con los currículos impuestos por las instituciones, ya sean nacionales (ministerios) o transnacionales (entidades financieras), que esquematizan contenidos homólogos y aplican pruebas taxativas, desconociendo el conocimiento y las diversidad de los pueblos latinoamericanos.

\section{REFERENCIAS BIBLIOGRÁFICAS}

ESCOBAR, ARTURO. (2003). Mundos y conocimientos de otro modo. El programa de investigación de modernidad/colonialidad latinoamericano. En: Tabula Rasa N. 1. Pp. 51-86. En línea: http://www.unc.edu/ aescobar/text/esp/escobar-tabula-rasa.pdf.

MIGNOLO, WALTER. (2001). Capitalismo y geopolítica del conocimiento. Buenos Aires: Ediciones del Signo.

TIRAMONTI, GUILLERMINA. (2005). La escuela en la encrucijada del cambio epocal. En: Educación y Sociedad. vol.26 no.92 Campinas Oct. 2005. En línea: http://www.scielo.br/ pdf/es/v26n92/v26n92a09.pdf.

TADEU DA SILVA, TOMAZ. (1997). Descolonizar el currículo: estrategias para una pedagogía crítica (dos o tres comentarios sobre el texto de Michael Apple). En Gentili, Pablo [comp.] (1997), Cultura, política y currículo. Ensayos sobre la crisis de la escuela pública, Buenos Aires, Losada, cap.3, pp. 64-78.

WALSH CATHERINE. (2000). Propuesta para el tratamiento de la interculturalidad en la educación. Lima: UNEBI-MED.

WALSH, CATHERINE. (2006). Interculturalidad y (de) colonialidad: diferencia y nación de otro modo. En: Livro da Academia da Latinidade. En línea: http://www.ram-wan.net/ restrepo/decolonial/18-walsh-interculturalidad20y20decolonialidad.pdf.

WALSH, CATHERINE. (2007). ¿Son posibles unas ciencias sociales/culturales otras? Reflexiones en torno a las epistemologías decoloniales. En: Revista Nómadas No. 26. Abril de 2007. Pp. 102-113. Bogotá: Universidad Central. En línea: http://www.ucentral.edu.co/ NOMADAS/nunme-ante/26-30/nomadas26/8-SON20POSIBLES20CATHERINE.pdf. 